\title{
SITUAÇÃO DA ENFERMAGEM NA DÉCADA DE 80 \\ - No chão, brotando para os anos 90 - \\ EXPERIÊNCIAS vividas e RESULTADOS obtidos na DIREÇÃO \\ de um Hospital Público - UFRJ, "num mundo em CRISE”.
}

Nébia Maria Almeida de Figueiredo*

Izabel Rosa dos Santos**

\begin{abstract}
RESUMO - Este trabalho é o resultado da experiência de Implantação de uma proposta inovadora sobre o assistir numa Instituição Pública dirigida por enfermeira, onde se desvenda os caminhos da enfermagem para os anos 90. O marco do trabalho é INTERDISCIPLINAR, num constante FAZER APRENDENDO, cuja administração é LEVE e FLEXÍVEL. A assistência é orientada por Projetos, onde estão contidos Programas que seguem a filosofia da REFORMA SANITÁRIA e está integrada no SISTEMA UNIFICADO DESCENTRALIZADO de SAÚDE.
\end{abstract}

ABSTRACT - This work is result of experience of implantation the innovation proposal this way to be present in Public Instituition nurse administrate, where to take off the bandage the way of nursing 90 years. The mark is interdisciplinarity work, in constant to do learning, which administration is light and flexible. The assistance is orienting by projects, where is contained programs who to follow the philosoply of "Reforma Sanitária", it is integrated in "System Unificated Descentralized of Health".

\section{INTRODUÇÃO}

Mais de sessenta anos se passaram desde que o Cientista CARLOS CHAGAS trouxe a Enfermagem Moderna para o BRASIL em 1926. Jamais desvalorizaria o feito e, muito menos acharia que as Enfermeiras Americanas que vieram para Implantar e Implementá-la, principalmente na Área de Saúde Pública, "NĀO" cumpriram com suas tarefas. Naquela época a Reforma Sanitária tão decantada e assegurada, na nova Constituição do Brasil de 1988, estava definida e sendo implantada.

No entanto, temos motivos, enquanto enfermeiros da década dos anos 80, para pensar que houve acidente de percurso na evolução da enfermagem, e alguma coisa aconteceu no decorrer do tempo. Caso contrário a enfermagem ainda hoje não estaria questionando coisas como: o que enfermeiro faz, qual o significado da enfermagem e da sua prática, porque a sociedade não nos reconhece etc.. VARGENS (1987) diz "que os enfermeiros estão em fase de afirmação e aceitação interna de nossos princípios e teorias, que estão em dúvida sobre seus conhecimentos científicos sua aplicabilidade e até mesmo de sua validade. Ao mesmo tempo que afirma que o 'agir' $e$ 'pensar' da enfermagem estão cada vez mais distantes um do outro".

A enfermagem moderna de CARLOS CHAGAS caminha de mãos dadas com a enfermagem antiga de ALFREDO PINTO (ambas muito atuais) numa época em que estamos em plena CRISE de identidade pessoal e profissional. $O$ enfermeiro está sendo checado por sua equipe e o entendimento de seu trabalho é confuso.

Sentimos durante 18 anos de trabalho um legado de nossos ancestrais profissionais, na própria pele que estamos precisando imediatamente REVER e/ou
RESGATAR o que não conseguimos fixar, como: Autonomia, Poder, Importância.

$\mathrm{O}$ relacionamento da enfermagem quer na prática profissional ou com a sociedade é marcada por contradições e incoerências. Mesmo assim, entendemos que ainda estamos escrevendo nossa história e 60 anos no tempo não significam nada para os historiadores.

O caminho para uma melhor posição e afirmação para os anos 90 existe, basta que as Escolas mudem seus currículos, e que saiam de uma visão patológica para uma visão fisiologica do assistir; que coloque na Sociedade enfermeiros "guerreiros" capazes de se adaptar num mundo em constante mudança, abertos, de centro com relação ao autoritarismo. Nesta década com certeza, a enfermagem está NO CH Ã̃ $\mathbf{O}$, numa década marcada por conflitos, numa necessidade de enraizar sua prática, buscar a direção, do sol para se reerguer, num solo íngrime que-é o sistema de saúde (sucateado, falido e desmoralizado) do Brasil.

Gerenciar e crescer nestas condições é muito difícil, definir seu signifivado muito mais, segundo CARVALHO (1988), “a preocupação com o significado das coisas tornou-se regra comum porque estamos vivendo uma crise sem precedente. $E$ dizer que o significado de uma coisa não é o mesmo que resolver as dificuldades de sua situação no mundo ou o problema que por ventura ou por hipótese, lhe diz respeito, definir o 'significado da assistência de enfermagem hoje' já é, por si, um problema".

Estar no chão também tem um outro significado, parece que caímos do cume em que fomos colocados em 1926 até mais ou menos a década de 40 (épocas de grandes prestígios) e estamos inertes, deitados. Levantar significa voar, perder o medo de largar o que está instituído e lhe parece seguro, enfrentar esta realidade de CRISE (da saúde, do mundo, das relações

\footnotetext{
* Diretora do Hospital Escola São Francisco de Assis da UFRJ. Professor Assistente IV da Escola de Enfermagem Anna Nery: Enfermeiro Supervisor do Hospital Universitário Gaffrée Guinle.

** Professor Assistente IV da Escola de Enfermagem Ana Nery, Assistente do Diretor do Hospital Escola Säo Francisco de Assis -RJ.
} 
humanas e da própria equipe de enfermagem).

Sair debaixo do poder, principalmente do médico e ficar do lado dele, significa não ter medo de errar e de se expor.

\section{JUSTIFICATIVA}

Relatar a experiência que estamos vivendo com enfermeiros que dirigem um Hospital da UFRJ é de grande importância não para nós, mas para toda a enfemagem brasileira. Esta realidade abre um precedente para que outros possam dirigir Instituições Públicas, cargo assegurado apenas para médicos.

Por entender que esta oportunidade tem mostrado um outro lado da enfermagem, que of iciosamente dirige hospitais, e mantém seu funcionamento, mas nã̃o lhe é reconhecido este direito.

Por perceber que os enfermeiros tem capacidade de se adaptar mais facilmente que os outros membros da equipe da saúde, e dizer que o mundo que estamos vivendo hoje tem nos obrigado a seguir outros caminhos, principalmente na área de administração.

\section{METODOLOGIA}

- Histórico sobre a reativação do Hospital Escola São Francisco de Assis - HESFA-UFRJ.

- Proposta de Trabalho e Implantação * Dificuldades e Facilidades

- Avaliação do Trabalho - Administração de Projetos * Resultados obtidos

\section{Histórico}

O Hospital Escola São Francisco de Assis HESFA da UFRJ foi reativado em fevereiro de 1988, por ocasião da calamidade instalada no Rio de Janeiro. A definição de sua proposta de trabalho partiu da Escola de Enfermagem Anna Nery - EEAN cujo conceito de Saúde é o adotado na 8ª Conferência Nacional de Saúde: "Ter Saúde não significa somente estar livre de doenças e sofrimentos físicos e psíquicos, mas, também usufruir das condições necessárias à concretização de uma vida digna, e que o direito à saúde inclua, a possibilidade de realização plena como ser humano".

O Hospital foi definido como Comunitário, dispondo de Unidades Assistenciais voltadas para Promoção, Tratamento e Reabilitação, os serviços são of erecidos através das seguintes unidades: Unidade de Internação - UI; Unidade de Semi-Internação - USI; Unidade de Cuidados Básicos - UCB e Unidade de Reabilitação - UR.

O HESFA tem quatro características que o defini como Unidade de Serviço diferenciado da rede pública de assistência à Saúde, a saber:

1 - Serviços Básicos e de Internação operados e gerenciados segundo a especificidade da área da enfermagem;

2 - Promoção do auto cuidado, recuperação e reabilitação de clientes e apoio aos familiares;

3 - Apoio a complementação de outras unidades de serviços hospitalares de maior complexidade;

4 - Desenvolvimento de atividades comunitárias como estratégia de ação.

\section{PROPOSTA DE TRABALHO E IMPLANTAÇÃO}

A proposta assegura que a filosofia da assistência segue a da REFORMA SANITÁRIA e está integrada no SISTEMA UNIFICADO e DESCENTRALIZADO DE SAÚDE.

O Cliente para a Unidade de Internação é aquele com as seguintes características: "Portadores de problemas de menor complexidade, que necessitem basicamente de assistência de enfermagem e de nutrição de alta qualidade", numa concepção de cuidados progressivos.

A teoria que respalda o trabalho é a do AutoCuidado e a assistência prestada nas unidades de SemiInternação, Cuidados Básicos e Reabilitação se orientam pelos Programas da Saúde da Mulher, Saúde do Adulto, Saúde da Criança, Saúde do Adolescente e pelos programas específicos ligados as doenças crônico-degenerativas, pulmonares, dermatológicas. (Anexo I)

\section{Em segundo lugar:}

O marco do trabalho é a INTERDISCIPLINARIDADE, cujos integrantes pertencem à várias carreiras, onde devem of erecer abordagens diversas a um dado problema. Uma assistência que deve estar voltada para um consenso comum, embora, as áreas de conhecimentos sejam diferenciadas, visando o seguinte objetivo: "Qualidade de assistência que deve ser prestada ao cliente, que deve ser visto como um todo individualizado e parte integrante de uma família e sua respectiva Comunidade, que deve participar dos trabalhos, discutindo, opinando e muitas vezes até decidindo que fazer"; cuja dificuldade maior foi assegurar o espaço de cada profissional no sentido do entendimento deste tipo de trabalho, onde devem preservar o modo peculiar de assistir de cada um, somando espaços para potencializar os benefícios destinados aos c iéntes. $\mathrm{O}$ trabalho conjunto resultou após vários embates, criando um novo poder - o do grupo.

A Interdisciplinaridade segundo CARVALHO (1983) que afirma "é preciso fazer alguma coisa para preservar a Unidade ou não, não há meio de se manter um todo, em sua inteireza, cuidando apenas de uma de suas partes, ou cuidando das partes separadamente. $\dot{E}$ preciso unir os elos, apertar as juntas, fortificar as ligaduras ou construir as pontes, e ainda apoiar o crescimento harmonioso das partes e a articulação mesmo entre elas".

Em terceiro lugar:

Talvez se ja a administração LEVE e FLEXÍVEL, como a proposta mais revolucionária na medida em que dá oportunidade de freqüentes mudanças, para adaptar ou corrigir o trabalho assistencial.

Uma proposta dinâmica, cujos comandos são em linha horizontal of erecendo oportunidade a todos.

No início parecia o próprio movimento "Braw niano", "confuso e desordenado", pois todos nós vínhamos de experiências centradas em organização es truturada, vertical e rígida. Os ortodoxos têm visto a experiência ceticamente, no entanto, se percebe que é um trabalho rico e capaz de enfrentar as crises do 
mundo de hoje, num trabalho que tem gerado interesse e satisfação no grupo, sem no entanto afirmar que é a totalidade.

O organograma do HESFA (anexo II) mostra a característica de lexeza, onde dispõe das seguintes áreas funcionais:

\section{1 - Coordenação de Serviços de Saúde \\ - Unidade de Internação \\ - Unidade de Semi-Internação \\ - Unidade de Cuidados Básicos \\ - Unidade de Reabilitação}

\section{2 - Coordenação Administrativa}

- Unidade de Documentação e Estatística

- Unidade de Finanças e Contabilidade

- Unidade de Recursos Humanos

- Unidade de Serviços Gerais

\section{3 - Coordenação de Apoio Técnico}

- Unidade de Laboratório de Análises Clínicas

- Unidade de Farmácia

- Unidade de Nutrição e Dietética

- Unidade de Imagens e Traçados Gráficos

- Unidade de Estudos e Pesquisa

Assim, no processo de organização interna do HESFA procurou-se solucionar as questões relacionadas à definição hierárquica, ao interrelacionamento das diversas funções e à integração necessária à consecução dos Objetivos do Hospital.

Definiu-se os níveis decisórios como superior e intermediário.

CONSEL HO DEL IBERATIVO - Órgão de deliberação superior responsável pelo funcionamento do HESFA, com atribuições relacionadas à determinação e aprovação de normas, promoção de acordos e convênios e demais iniciativas necessárias a coordenação adequada das ações implementadas pelo Hospital composto por autoridades da UFRJ com representatividade com as funções do HESFA, além de representantes do SUDS e da Comunidade.

DIREÇÃO DO HESFA - Composta pelo Diretor e seu assistente. Ao primeiro são delegadas atividades executivas, pelas autoridades componentes do Conselho Deliberativo. É o mais alto nível de supervisão, sendo responsável pelo funcionamento de todas as atividades técnicas, administrativas e de apoio do Hospital.

CONSEL HO COMUNITÁRIO - Composto por representantes da Comunidade organizada, segundo critérios da própria comunidade, por representantes de cada Unidade da Coordenação de Serviço e Saúde e pela Direção do Hospital, aos quais cumpre discutir as diretrizes operacionais relacionadas à assistência.

CONSEL HO TÉCNICO CONSUL TIVO - Órgão colegiado composto pelos coordenadores das três áreas funcionais que compõem o Hospital e da Assessoria de Programas e Projetos com o objetivo de definir operacionalmente as diretrizes baixadas pelo Conselho Deliberativo a fim de adequar a programação de atividades com a proposta orçamentária, segundo as prioridades estabelecidas.

ASSESSORIA DE PLANEJAMENTO - Órgão responsável pelo planejamento e acompanhamento do desempenho das Unidades Organizacionais a partir da implantação do Sistema de Controle de Custo e formalização da proposta orçamentária do HESFA.

ASSESSORIA DE PROGRAMAS E PROJETOS - Área responsável e voltada para análise das atividades Técnico-Científicos com a atividade de emitir parecer, acompanhar e avaliar Programas e Projetos aprovados e implantados nas Unidades da Coordenação de Serviço de Saúde.

CENTRO DE PROCESSAMENTO DE DA DOS - Órgão responsável e voltado para a sistematização e consolidação das informações geradas nas Unidades organizacionais, assim como pela prestação de serviços de informações à Assessoria de Planejamento e às Unidades organizacionais.

COORDENAÇÃO ADMINISTRATIVA Área de competência voltada para a promoção e manutenção do funcionamento do Hospital, desenvolvendo, através de suas unidades as ações necessárias ao planejamento, coordenação, supervisão, execução e controle das atividades nas áreas de pessoal, documentação e estatística, finanças e contabilidade, compra, manutenção, transporte, zeladoria, rouparia, telefone e vigilância.

COORDENAÇÃO DE APOIO TÉCNICO Área destinada a of erecer apoio técnico à área finalística do HESFA, através das unidades de laboratório de análises clínicas, farmácia, nutrição e dietética, diagnóstico por imagens e traçados gráficos e esterelização de material.

COORDENAÇÃO DE SERVIÇO DE SAÚDE - Área finalística do HESFA, que concentra as atividades de atendimento de clientes nas Unidades de Internação, Semi-Internação, Reabilitação e Cuidados Básicos de Saúde e de promoção científica.

Esta estrutura tem criado muita pessão por parte dos conservadores os quais não se veem dentro de uma estrutura de poder, na verdade quem direciona o que fazer no HESFA é a clientela. VAIL (1950) diz que "quando uma sociedade é atingida por uma onda de mudança, ver-se freqüentemente forçada a reavaliar suas convicções, e, ela pode descobrir que determinadas conviç̧ões antigas são irrelevantes ou se tornam superadas e chama a atenção para o perigo de idéias enferrujadas, as quais são mais perigosas do que máquinas obsoletas. A criação de um conselho comunitário incomoda e nos obriga a despadronizar a assistência, fazendo com que esta possa ser globalizada e individualizada, pois é a partir dos desejos da comunidade que as ações são implantadas. Esta administração parece ser uma revolução no modo de trabalhar no HESFA. A industrialização trouxe a produção em massa, o mesmo ocorrendo com o sistema de saúde, que agora deve sofrer uma mudança total se a Reforma Sanitária for implantada na íntegra".

TOFFLER (1985) provavelmente é o escritor que mais tem certeza de que o mundo está em ebulição e que as mudanças são inevitáveis nos tempos de hoje. $\mathrm{E}$ afirma que as empresas menos flexíveis terão dificuldades de se adaptar e "que confiar na tradição pode ser inteiramente apropriado num meio familiar ou estável, talvez se torne uma atitude desastrosa num meio novo, em rápida transformação, no qual os próprios problemas sâo novos e de mudança rápida, onde os indivíduos são para o sistema, provém cada vez mais de grupos populacionais, que até recentemente estavam representados de uma maneira mínima na força de tra- 
balho ou sofreram mudanças radicais de perspectiva durante a última década".

As mudanças se refletem na enfermagem, na comunidade que assistimos e a construção do trabalho no HESFA considera todas estas questões.

O próprio trabalho é o resultado de um projeto inicial, que também estamos administrando como o projeto maior que deu origem aos seguintes PROJETOS E PROGRAMAS:

Para implantação e administração do Projeto HESFA foram realizados 38 (trinta e oito) encontros para discutir a proposta onde foram gastas 152 (cento e cinqüenta e duas) horas.

Agora um ano depois estamos desenvolvendo seminários para discutir o entendimento sobre interdisciplinariadade e administração flexível, para verificar se a prática e a teoria estão andando juntas.

Os projetos são analisados através da Assessoria de Programas e Projetos - APP composto de cinco profissionais da área da saúde. A implantação e acompanhamento deles está sob a responsabilidade do coordenador de cada programa e/ou projeto que faz o feedback com a APP.

\section{AVALIAÇÃ O DO TRABALHO DURANTE OS 12 MESES DE IMPLANTAÇÃO DA PROPOSTA}

\section{Resultados obtidos}

Profissionais - ainda hoje há necessidade de encontros freqüentes para discutir a questão da interdisciplinaridade e definir ações e espaços, freqüentemente há necessidade de rever posições e ajustar as relações inter-pessoais. Mas, de uma maneira geral, todos estão integrados nos Projetos e Programas, os quais são responsáveis pelo sucesso ou fracasso dos mesmos.

Cada Projeto define sua finalidade e o tipo de cliente que vai assistir.

\section{IENTEL A}

\section{NA UNIDADE DE INTERNAÇÃO - U.I.}

Sua proposta de porte é de 160 leitos, no entanto, devido as condições em que se encontra o Hospital por ter estado fechado há dez anos (está sendo restaurado) apenas 40 leitos são of erecidos às Instituições, e, de março/88 a junho/89 recebemos 420 clientes, em fase crônica, portadores de deficiências motoras, em fase terminal, que necessitavam de orientação para o auto cuidado, orientação familiar para a alta e visitas domiciliares para diagnóstico da ambiência e possibilidades de retorno ao lar.

É importante informar que $80 \%$ destes clientes necessitavam basicamente de assistência de enfermagem onde suas ações estão relacionadas à:

- conforto e higiene

- orientação para o auto-cuidado (auto medicação, glicosura, auto aplicação de insulina, cuidados com o corpo, auto alimentação por sondas etc.)

- prevenção de escaras e pneumopatias (através de mobilizações)

- administração de medicamentos

- reeducação vesical

- atividades recreativas
- visita à família durante a internação e depois da alta do cliente.

- orientação à família para participação da assistência.

As outras ações estão distribuídas entre os outros profissionais, no entanto, há de se ressaltar que em algumas situações o percentual muda, principalmente para a medicina, que depois o devolve para a enfermagem, a qual tem absorvido toda sua força de trabalho.

A Equipe se reúne semanalmente para ajustar e definir suas ações, delimitar espaços e criar critérios de assistir.

\section{NA UNIDADE DE CUIDADOS BÁSICOS -} U.C.B.

Os trabalhos nesta Unidade são orientados pelos seus Programas, num trabalho rico em experiências tanto com a própria equipe ou com a comunidade que nos procura para resolução de seus problemas.

É nesta Unidade que os profissionais da área da saúde atuam de forma mais integrada e isoladamente quando cada profissão entende que sua ação em determinado momento deve ser individualizada.

A Unidade discute freqüentemente suas dificuldades e a tomada de decisão emana do grupo. Suas ações são dirigidas de dentro para fora numa busca constante de assegurar e forçar o sistema de saúde na questão de contra-referência.

A sua coordenação está a cargo de uma enfermeira e o seu trabalho maior é equilibrar e fortalecer as relações, assegurando os espaços de cada um e da enfermagem mais precisamente.

$O$ trabalho de SALA de ESPERA foi iniciado por psicólogos, mas entendido como uma ação interdisciplinar, e a medida que as discussões vão ocorrendo a equipe vai sentindo necessidade de maior integraação, de chegar mais, de unir suas forças e fortalecer seus laços. Um trabalho premeado por muits dificuldades e muitos conflitos. Apesar disto a UCB atendeu até agora $8.459^{\circ}$, cujas consultas ficaram assim distribuídas: (Anexo III).

A autonomia da enfermagem nas questões de promoção da saúde a nível primário, tem sido questionada, checada apesar da lei do Exercício de 1986.

$\mathrm{Na}$ verdade a assistência de enfermagem parece uma questão de muitos, e, todos parecem "pensar" que sabem o que é a enfermagem e o que ela não é.

Entendido e compreendido por nós que a percepção dos demais membros da equipe de saúde, está relacionada a sua formação profissional onde a enfermagem é difundida como uma profissão "periférica" e que obedece as ordens médicas, na verdade ela é autônoma e liberal.

PAIM (1977) tem uma posição acerca deste assunto, onde descreve que a enfermagem é indefinida quanto ao que ela é, e o que não é, e, que nos aceitamos isso como algo inseguro e gerador de grande ansiedade em todo o grupo profissional, principalmente quando "outros" questionam quanto a utilidade desta profissão, pelo menos com formação de nível superior; e diz: "Quanto mais isso ocorre, mais devemos estar cientes de que o fato de estar sempre em busca de uma definição, significa para todos nós um continumpor que indica que zelamos por questionar e revalidar as nossas concepções, e isso traduz que nosso perfil 
precisa estar ajustado ao que deseja de nós, a sociedade brasileira".

Os conflitos inter-pessoais e profissionais têm sido uma das grandes dificuldades para um maior crescimento do grupo, que tem dificuldades individuais de entender a dinâmica administrativa e o trabalho interdisciplinar. Um trabalho que desestabiliza poderes instituídos, força, numa discussão em mesmo nível, que obriga as discussões em grupos maiores, que demora a decidir as coisas, porque todos devem falar e opinar principalmente, quando há participação da Comunidade.

Nós enquanto gerenciadores deste grande Projeto, temos sido obrigados a assumir uma função catalizadora e a tentar fazer com que a Proposta crie raízes nas Comunidades Interna e Externa do HESFA, num momento em que a UFRJ vive a sua maior CRISE, política, social e econômica. Nós estamos neste' roldão e o nosso dia-a-dia tem sido gerenciar:

a) uma Instituição sem recursos e um projeto inovador;

b) um grupo jovem e em conflito pela própria característica do trabalho;

c) um grupo formado por todos os membros da equipe de saúde, sendo nós enfermeiros;

d) as relações entre as unidades de Ensino da UFRJ;

c) a presença do HESFA nos órgãos de decisão da UFRJ quando somos jovens em termos de maior experiência nestes colegiados;

f) nossas emoções e sentimentos, enquanto enfermeiros, para que não sejamos tendenciosos com relação aos demais profissionais numa atitude de superproteger a enf ermagem;

g) a busca de alternativas às outras Instituições de Saúde avançando nas discussões e assumindo compromissos para que o trabalho do HESFA se ja entendido;

h) os problemas que emanaram da Fiscalização de Medicina dificultando o nosso trabalho por sermos enfermeiros, questionamentos um pouco amenizado neste momento, mas não esquecidos;

i) gerenciar os desagrados ouvidos na Congregação da Medicina da UFRJ por sermos enfermeiros, mas sanados de forma significativa e positiva no final da mesma;

j) a recusa de algumas Instituições em não nos atender por sermos enf ermeiros;

l) as dificuldades de um maior entendimento entre os nossos próprios colegas de unidade-EEAN;

m) discutir os convênios que foram assinados com o HESFA, numa linguagem desconhecida por nós e num trabalho conflitante devido a própria política de Saúde, quando a nossa opção foi pelo SUDS.

Até o momento, ou mais precisamente, partici- pamos nestes 16 meses de 711 reuniōes, para discussão de todo tipo de questão com as mais variadas autoridades e Instituições.

\section{CONCLUINDO:}

Este é um trabalho que não pode ser concluído neste momento, estamos relatando uma experiência nova vivida por dois enfermeiros que se aventuraram a implantar uma Proposta de trabalho, com um grupo originariamente não acostumado a dirigir (médicos, psicólogos, assistentes sociais, fisioterapeutas, fisiatras, nutricionistas, farmacêuticos e profissionais administrativos), numa amplitude muito maior onde administração de assistência de enfermagem é apenas a parte de um todo.

Provavelmente, é um trabalho que não tem fim, as demais Unidades assistenciais não discutidas isoladamente estão sendo implantadas, construídas e a cada momento estamos adaptando de acordo com a solicitação e demanda de nossa Comunidade.

Paralelamente podemos assegurar que apesar de ser um trabalho extremamente difícil, é bastante excitante, gratificante, principalmente em descobrir que apesar de todos os conflitos, os profissionais do HESFA correspondem as expectativas de sua proposta. Que cada um em particular renova suas ações, moderniza e despadroniza seu assistir. Um trabalho que só é possível porque é o resultado do esforço de todos, e que os conflitos têm nos levado ao consenso, tem criado laços, tem fortalecido nossos problemas afetivos e interpessoais.

\section{BIBLIOGRAFIA}

1 ALVES, S. M. et alii - En'fermagem. Contribuição para o cálculo de recursos humanos na área. Ministério da Previdência e Assistência Social INAMPS. 1 ${ }^{\mathrm{a}}$ ed. Rio, 1988.

2 ALVIM, E. F. \& MATOS, A. V. O Hospital como centro de irradiação das atividades de saúde pública. Rio de Janeiro, Rev. Bras. Enf. 1962:362-371.

3 APOSTEL, L. et alii. Interdisciplinariedad - Problemas de $1^{\text {a }}$ ensenãnza y de la investigacion en las Universidades. Centro para a Investigação e Inovação do Ensino. Universidade de Paris, 1975.

4 AZEVEDO, D. O. et alii. Escola aberta para a comunidade. Tema livre - $36^{\circ}$ Congresso Brasileiro de Enfermagem Belo Horizonte, 1984.

5 BERGO, M.C.N.C \& CAMARGO, V.O.C. Sistema de Hospital-Dia. Uma experiência na assistência de enfermagem - Hospital Albert Einstein, São Paulo, Tema Livre - 35\% Congresso Brasileiro de Enfermagem - São Paulo, 1983.

6 CARVALHO \& CASTRO - Vilma de e Ieda Barreiro e Das partes necessárias à articulação da Graduação com a Pós-Graduação na Escola de Enfermagem Anna Nery ano I, n' I - $1988-R J$.

7 CIBOTTI, R. Notas sobre $1^{\text {a }}$ seleción de tecnologias apropriadas em los servicios de salud. OPAS/OMS. Simpósio sobre Tecnologia Apropriada para Salud - Washington, 1980.

8 CHIAVEnAtO, I. Teoria Geral da Administração. Abordagens prescritivas e normativas da administração - $3^{\text {a }}$ ed., São Paulo. MG Graw - Hill, 1987. 
9 CUKIERMAN \& DINSMOR, Zigmundo Salomão e Paul Campbell - Administraçāo de Projetos, caracterizaçāo e Problemática - Editora Guanabara - RJ - 2ª Ediçāo.

10 DRUCKER, Peter F. - Administração em Tempos Turbulentos - Editora Biblioteca Pioneira de Administração e Negócios. 1980 - São Paulo - 2ª edição.

11 FERNANDES, J.D. Contribuição da Equipe Multiprofissional nas Açōes de Saúde - Mito ou realidade? Brasília - Rev. Brasileira de Enfermagem: 175 - 181 abr./jun. 1981.

12 FALKEMBACH, E.M.F. \& BELATO, N. O. Planejamento participativo Petropolis: Vozes, 1987.

13 GALIZA, M. C. et alii - Enfermagem e Farmácia Clínica Nova Integração profissional. Tema Livre - 35a Congresso Brasileiro de Enfermagem - São Paulo. 1983.

14 GARCIA, R. A. et alii - Unidade de Semi-Internação do Centro Geral de Pediatria em Belo Horizonte, Tema Livre, 36을 Congresso Brasileiro de Enfermagem - Belo Horizonte, 1984.

15 GONÇALVES - Emesto Lima - Administração de Saúde no Brasil - Biblioteca pioneira de Administração e Negócios - São Paulo, 1985.

16 NAKAMAE - Dejair Daniel - Novos Caminhos da Enfermagem - São Paulo: Cortez, 1987.
17 PAIM - Lygia - Questōes e Mitos Acerca da Assistência de Enferrnagem - Rev. Brasileira - 1977 - Vol. 02.

18 REIS, I. B. et alii - Cuidado Progressivo ao Paciente: Implantação e sistematização - Tema Livre - 35 Congresso Brasileiro de Enf ermagem, São Paulo, 1983.

19 RESENDE, A. L. M. Saúde: Dialétetica do Pensar 4 e do Fazer. Corez, São Paulo, 1986. 8: CONFERÊNCIA NACIONAL DE SAÚDE - Relatório Final - Brasília, 1986.

20 SCHORR, T. Los componentes lógicos en la gestión de transferência entre Tecnologia Apropriada Y Sociedad. OPS/OMS. Simpósio sobre Tecnologia Apropriada para Salud. Washington, 1980.

21 SILVA, R. B. B. et alii, Projeto de permanência conjunta. Hospital da Criança Santo Antonio - Porto Alegre - Tema Livre - 360 Congresso Brasileiro de Enfermagem - Belo Horizonte - Agosto, 1984.

22 TOFFLER, Alvin - A Empresa Flextvel - Editora Record 1985, RJ. 3a Ediçāo.

23 VARGEN, Otávio Muniz de Castro - Em Busca de um espaço - Revista Paulista de Enfermagem - São Paulo, 1987.

24 WORLD HEALTH ORGANIZATION - Role of Hospitals in Ambulatory and Domiciliary Medical Gare. Technical Report Series, no176 Genebra, 1959.

PROBLEMAS MAIS FREQÜENTEMENTE DETECTADOS NA U.C.B.

a) Em adultos:

- Carcinoma

- Diabetes melitus

- Hipertensão Arterial

- Seqüela de AVC

- Problemas de pele

- Problemas Gástricos

- Obesidade

b) Em crianças:

- Dermatose

- Escabiose

- Verminose

- Pediculose

- Intoxicação alimentar

- Problemas Gastro-Intestinais

- Problemas Respiratórios. 
A N E X O II

\section{ORGANOGRAMA}

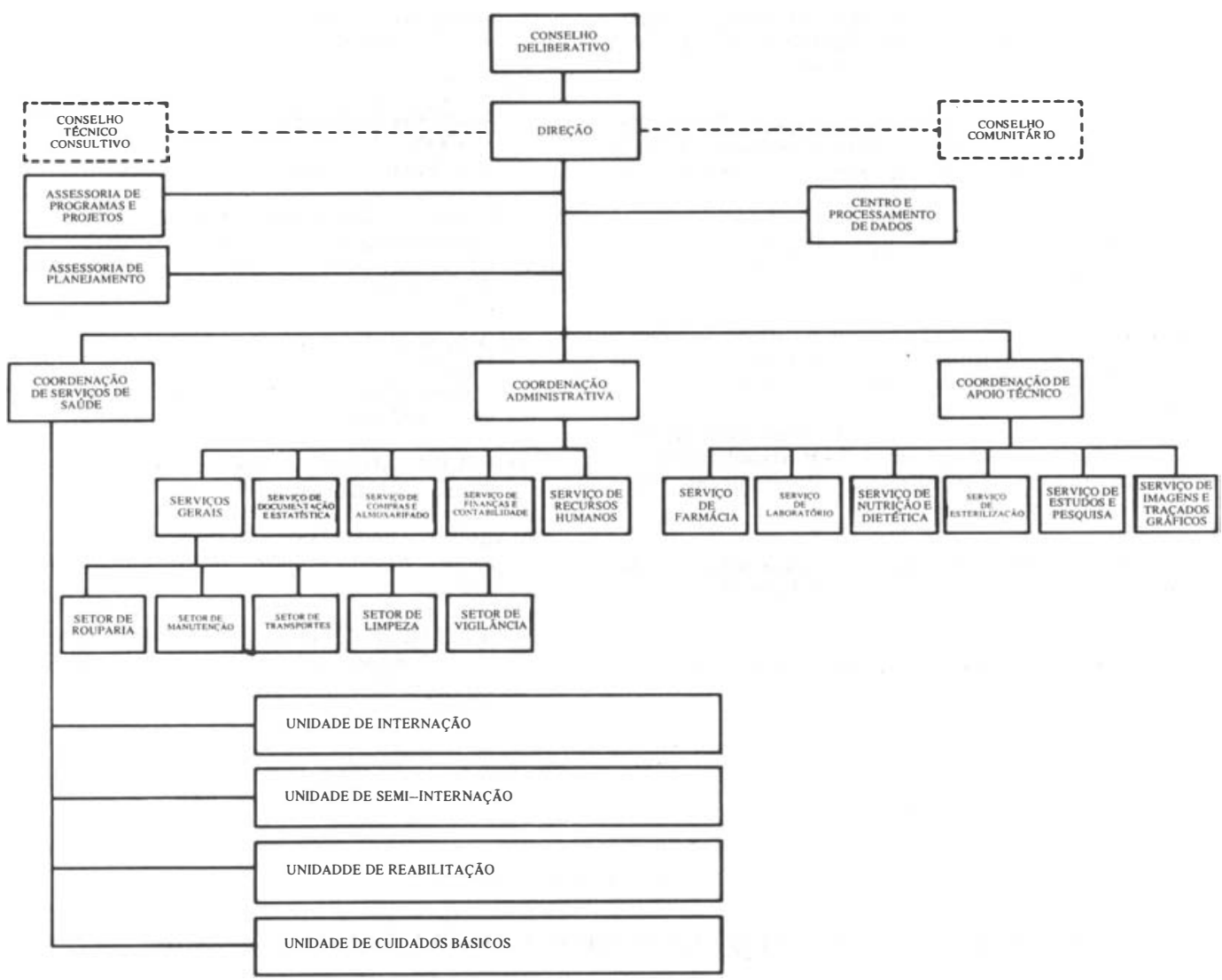

A N EXO III

ATENDIMENTOS REALIZADOS NA UCB E UI DE MAIO/88 A MAIO DE 89

\begin{tabular}{l|c|c}
\hline \multicolumn{1}{c|}{ MESES } & CONSULTAS & ALT AS \\
\hline Abril & - & 11 \\
Maio & 09 & 20 \\
Junho & 65 & 21 \\
Julho & 203 & 21 \\
Agosto & 274 & 25 \\
Setembro & 363 & 18 \\
Outubro & 290 & 17 \\
Novembro & 643 & 31 \\
Dezembro & 695 & 14 \\
Janeiro & 1.117 & 33 \\
Fevereiro & 1.016 & 41 \\
Março & 993 & 20 \\
Abril & 1.051 & 26 \\
Maio & 740 & 294 \\
\hline T O T A L & $8.459 *$ & \\
\hline
\end{tabular}

* 3957 - Foram Consultas realizadas pela enf ermagem $e$ 2095 - atendimentos realizados na triagem. 